\title{
1840 - die Blütezeit der plastischen Chirurgie: Krankheitskonzepte und ihr Einfluss auf die chirurgische Praxis
}

\author{
1840 - The Time of Prosperity of Plastic Surgery: \\ Concepts of Disease and their Influence on Surgery
}

Autor

Institut

\section{L. Geiges}

Oberarzt Dermatologische Klinik Universitätsspital Zürich/Schweiz

Konservator Moulagenmuseum Zürich/Schweiz

Wissenschaftlicher Mitarbeiter Medizinhistorisches Insitut der Universität Zürich/Schweiz

\section{Bibliografie}

DOI $10.1055 / \mathrm{s}-0029-1214808$ Akt Dermatol 2009; 35: 271-274 @ Georg Thieme Verlag KG Stuttgart · New York ISSN 0340-2541

Korrespondenzadresse Dr. med. Michael L. Geiges Facharzt für Dermatologie und Venerologie Medizinhistorisches Institut der Universität Zürich Hirschengraben 82 8001 Zürich

Schweiz michael.geiges@mhiz.uzh.ch

\section{Zusammenfassung \\ $\nabla$}

Um 1840 publizieren die Dresdener Chirurgen Eduard Zeis und Friedrich August von Ammon Bücher zur plastischen Chirurgie und wähnten sich am Höhepunkt dieser Disziplin. Die großen Errungenschaften, welche die Chirurgie grundsätzlich veränderten, die Anästhesie und Asepsis, wurden allerdings erst entdeckt, nachdem Zeis

\section{Einleitung \\ $\nabla$}

Schaut man sich nach den Anfängen der plastischen Chirurge um, dann stößt man schnell auf die Dresdener Chirurgen Friedrich August von Ammon (1799-1861) und Eduard Zeis (18071868) sowie den „Vater der plastischen Chirurgie“, den Berliner Chirurgen Johann Friedrich Dieffenbach (1792-1847). Im Schlusswort seines durch die Medizinische Gesellschaft zu Gent preisgekrönten Buches „Die Plastische Chirurgie“ von 1842 [1] schreibt Friedrich August von Ammon begeistert, ganz im Sinne von Zeis [2] und Dieffenbach [3]:

„So gewährt die plastische Chirurgie, von alten Vorurtheilen befreit, und kühn und kräftig gehandhabt, jetzt große Resultate, [...]“

Folgt man der Geschichte eines technischen Fortschrittes, muss der Zeitpunkt erstaunen, zu dem diese Aussage gemacht wurde, denn erst vier Jahre später, im Jahr 1846, wird William Morton (1819-1868) in Boston die Äthernarkose demonstrieren, und erst über 20 Jahre später im Jahr 1867 publiziert Joseph Lister (1827-1912) sein antiseptisches Carbolspray-Verfahren, das zu einem Rückgang der gefürchteten Wundinfektionen führt. Waren etwa die Entwicklungen in der Schmerzbekämpfung und in der Entzündungsprophylaxe nicht Ursache, sondern vielleicht gar Folge des schon vorher stattgehabten Aufschwunges der Chirurgie, auch der plastischen seine Ansichten über „die Glanzperiode der plastischen Chirurgie“" publiziert hatte. Dies deutet darauf hin, dass die fortschrittsgeschichtliche Darstellung Ergänzungen benötigt. Insbesondere die sich wandelnden Krankheitskonzepte und die Bedeutung der Kosmetik verdienen eine größere Beachtung, da sie einen direkten Einfluss auf den Denkstil der Chirurgen hatten.

Chirurgie? Warum wurde bereits in der ersten Hälfte des 19. Jahrhunderts ein Höhepunkt in der Entwicklung der Chirurgie wahrgenommen? Erklärungen findet man in den Hauptwerken der oben erwähnten Chirurgen [1 -3].

\section{Die Geschichte der Rhinoplastik \\ $\nabla$}

Der Schwerpunkt und Ausgang der plastischen Chirurgie bildete die Rhinoplastik, gefolgt von anderen Operationen wie die Chiloplastik, Blepharoplastik, Canthoplastik, Otoplastik usw.

Früheste Spuren zur Rhinoplastik finden sich in Indien. Ob in der Antike das chirurgische Wissen und Können für diese Operation vorhanden war, ist schwer zu ermitteln und bleibt vorläufig unklar. Ab der Mitte des 14. Jahrhunderts tauchen in Europa erstmals Berichte über die Rekonstruktion von Nasen aus dem Oberarm auf. Der berühmte Französische Chirurg Ambroise Paré (1510 - 1590) behauptete im Jahr 1575, die Nase müsse in die Muskulatur des Oberarmes gesteckt werden, bis das Fleisch wieder an der Nase anwachse. Er schrieb aber auch, dass er selbst diese Operation nie gemacht habe und empfiehlt stattdessen den Einsatz von Epithesen, wie sie auch in seinem Werk abgebildet sind.

Die bekannteste Beschreibung einer Rhinoplastik stammt vom Bologneser Professor der Anatomie und Medicin, Gaspare Tagliacozzi (1545-1599). 
Er war als Nasenrestaurator berühmt, hat die Operation offenbar häufig durchgeführt und sie in seinem zweibändigen Werk „de chirurgia curtorum per insitionem“ 1597 in Venedig publiziert [4]. Er war zwar nicht, wie er behauptete, der Erste, der diese Operation durchgeführt hatte, aber er war der Erste und Wichtigste, der die Technik dank des Buchdruckes breit bekannt machte - ganz im Gegensatz zu anderen Chirurgen seiner Zeit, die ihr Können möglichst geheim hielten. Nur wenige haben Tagliacozzi nachgeahmt. Einzelne solcher Operationen sind im Sinne von Heldentaten überliefert. Spätere Autoren bezeichnen die Rhinoplastik als schmerzhaft, gefahrvoll und unnötig. Lieber werden Epithesen aus Silber oder Holz empfohlen. Immer fantastischere Geschichten über die immer sagenhaftere Rhinoplastik tauchen auf, wie z.B. ein Bericht aus dem Jahr 1682 über einen Nasenersatz durch die Haut eines anderen Menschen: Die Nase sei abgefallen, als der Spender gestorben sei. Schließlich wurde im 18. Jahrhundert die ganze Publikation von Tagliacozzi in Zweifel gezogen. Niemand hätte mehr den Mut gehabt, die Befunde auszuprobieren. Fast 200 Jahre lang findet sich kein konkreter Bericht mehr über die Durchführung eines chirurgischen Nasenersatzes. Die Behauptung, dass im 17. und 18. Jahrhundert wegen der Zunahme der Entstellungen durch die Syphilis die Rhinoplastik vermehrt praktiziert worden sei, wurde wahrscheinlich erst im Nachhinein in diese Periode hineinprojiziert.

1794 erregt ein Artikel aus den britischen Kolonien in Indien im „Gentlemen's Magazine“ in London, geschrieben von einem unbekannten Autor, vermutlich dem englischen Chirurgen Coly Lyon Lucas, über „the very curious, and, in Europe unkown chirurgical operation“ die Aufmerksamkeit der Chirurgen. Es brauchte offenbar, wie Zeis 1838 schreibt, „den barbarischen Einfluss des Ostens“, um die Rhinoplastik wieder aufleben zu lassen. In der Folge versuchten Chirurgen die Patienten sowohl nach der indischen als auch nach der italienischen Methode von Tagliacozzi zu behandeln. 1818 publizierte der Berliner Chirurgieprofessor Carl Ferdinand von Graefe (1787-1840) in einer Monografie eine von ihm nach dem italienischem Vorbild entwickelte deutsche Methode. Er benützte bereits im Titel erstmals den Begriff „Rhinoplastik“, von dem dann die Bezeichnung „plastische Chirurgie“" abgeleitet wurde. Schließlich setzte sich dieser Name gegen die französische Nomenklatur „l'Autoplastie“ durch. Zeis definiert die Fachrichtung so: „Die plastische Chirurgie ist derjenige Theil der operativen Chirurgie, welcher sich mit dem organischen Wiederersatze zerstörter Theile beschäftigt.“

Scheinbar waren die Animositäten zwischen Frankreich und Deutschland auch eine Triebfeder zur Publikation der deutschen Erfahrungen, denn Zeis erwähnt die französischen Chirurgen Martinet, Jobert, Labat und Blandin und schreibt: „Ungerecht gegen das Ausland, wie ihre Landsleute gewöhnlich sind, möchten uns die letzteren beiden (Labat in seiner Rinoplastie, Blandin in der Autoplastie) glauben machen, dass Frankreich das Land sei, wo die plastische Chirurgie gedieh, und zu ihrer jetzigen Grösse emporwuchs. " Es sei daher umso wichtiger, mit seiner Publikation zu zeigen, dass es eigentlich die Deutschen waren, welchen dieses Lob gebührt.

\section{Technik der Rhinoplastik}

$\nabla$

Die indische Methode mit einem heruntergeklappten Stirnlappen wurde eher abgelehnt, da sie zu zusätzlichen Vernarbungen und Wunden mit Zerstellungen am Kopf führte ( $\bullet$ Abb. 1). Hier

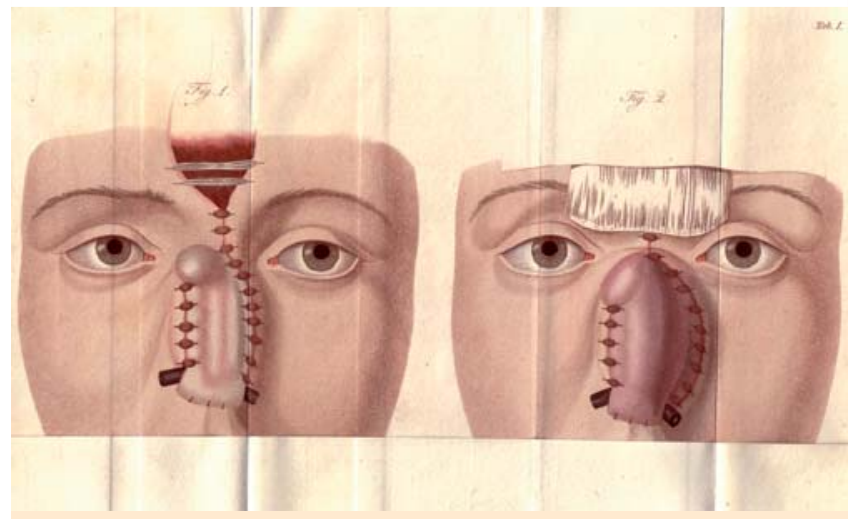

Abb. 1 Indische Methode von Zeis. Kupfertafeln zur Bildung einer Nase aus der Stirnhaut [1].

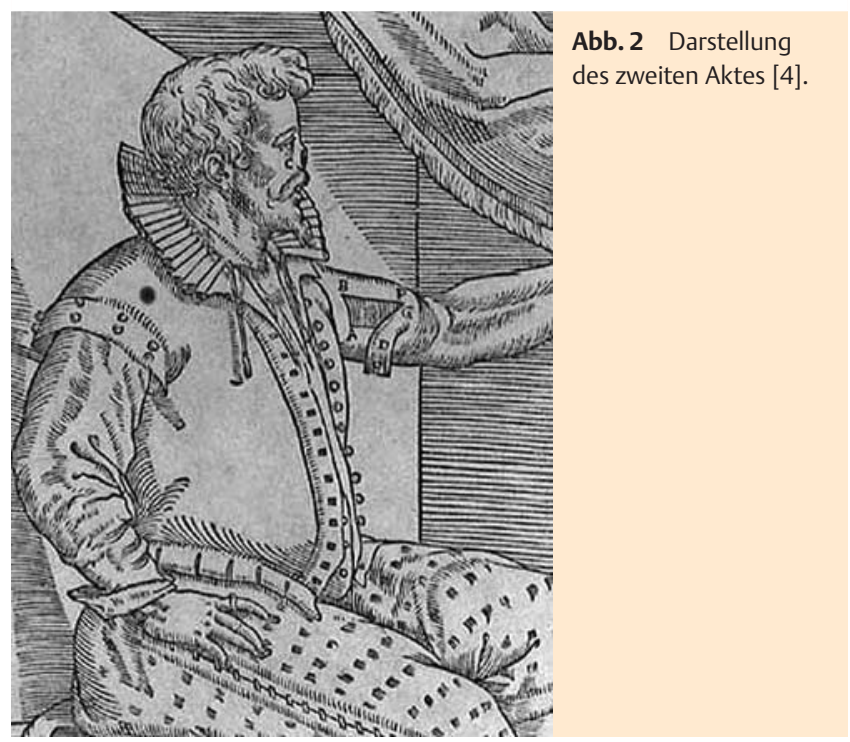

sei kurz die italienische Methode vorgestellt, die dann durch von Graefe zur deutschen Methode modifiziert wurde. Nach Tagliacozzi [4] beinhaltet sie sechs Akte:

1. Ablösung der Haut an der Vorderseite des Oberarmes und Unterlegung mit einem Leinenlappen

2. nach 14 Tagen Ablösung der ersten Hautbrücke ( $\bullet$ Abb. 2)

3. Der Lappen muss reifen. Er darf nicht zu viel Saft, aber auch noch nicht zu wenig Saft haben - er sollte, verglichen mit dem menschlichen Leben, das „männliche Alter“ erreicht haben, was in der Regel nach etwa 14 Tagen der Fall war. Dann erfolgte die Auffrischung des Nasenstumpfes, das Zurechtschneiden des Lappens und das Annähen.

4. Nach spätestens 20 Tagen wurde der Lappen vom Arm getrennt $($ Abb. 3).

5. Modellierung der Nase mit Binden

6. Anheften des Septums

Die Nachbehandlung zur Formung der Nase mithilfe von Nasenschienen dauerte dann noch mindestens zwei weitere Jahre. Neben der chirurgischen Technik waren humoralpathologische Überlegungen nötig, wie die Berücksichtigung der Jahreszeit zur Verhinderung des schlechten Eiters und zur richtigen Wahl des Verbandes. Bei schlechtem Wetter musste grundsätzlich jeweils zwei Tage länger gewartet werden, bevor zum nächsten Akt übergegangen werden konnte. In der Nachbehandlung waren ab- 


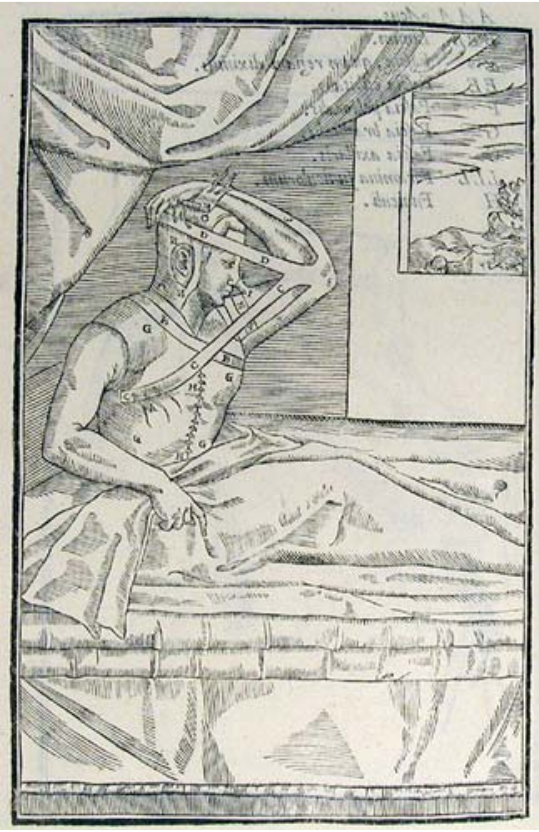

Abb. 3 Abbildung des Verbandes nach dem dritten Akt [4].

führende Mittel angezeigt und Aufregungen des Gemütes zu vermeiden. Neben der Ausbildung zusätzlicher Entstellungen war das Hauptrisiko die Gefahr von schwerem Wundbrand.

\section{Indikationen zur Rhinoplastik}

Die häufigste Indikation war der traumatische Verlust der Nase. Weiter wurden Syphilis, Skrofulose, Herpes und ganz neu auch Krebs als Indikationen diskutiert. Im Krankheitsverständnis um 1840 waren Hauterscheinungen immer noch primär Ausdruck einer Dyskrasie. Bei jeder Indikation musste geklärt sein, „in wie weit es nothwendig sei, vor der Ausführung plastischer Operationen vorhandene Dyskrasien im Körper zu tilgen“ [2].

Die Syphilis musste mit Quecksilber behandelt sein und so genannte herpetische Leiden vorher getilgt werden. Die Skrofulose, eine komplexe Problematik von Veranlagungen und Krankheitssymptomen, wurde unterschiedlich beurteilt und war nach Dieffenbach eine Kontraindikation.

Ein interessanter Wandel zeichnete sich bei der Chirurgie von Krebs oder Szirren ab. Mitte der 1830er-Jahre hatte Martinet de la Creuse Beiträge publiziert, in welchen er die Theorie aufstellt, dass das Karzinom dadurch zu heilen sei, dass man es rasch exstirpiere und die Wundfläche mit einem gesunden Hautlappen bedecke. Damit rüttelte er am Dogma, dass man „jedwelche Kachexie, gleichviel ob die vorhandene Verstümmelung von ihr abhängig sei oder nicht, zuvor aus dem Körper des Kranken entfernen müsse, bevor man an seine Heilung durch plastischen Wiederersatz denken dürfe“ [2]. Da die Ursache und das Wesen der Karzinome als unbekannt galten, wurden sie bis dahin zu den Kontraindikationen für die plastische Chirurgie gerechnet. Martinets Hypothese wurde mit vorsichtigem Enthusiasmus aufgenommen, denn sollte er Recht haben, würde sich ein riesiges neues Gebiet für die plastische Chirurgie eröffnen.

\section{Die Medizin als Naturwissenschaft}

\section{$\nabla$}

Was war der Hintergrund dafür, dass der Artikel im „Gentleman's Magazin“ der Idee der Rhinoplastik zu neuer Aufmerksamkeit verhalf und die alte italienische Methode wieder studiert und praktiziert wurde?

Seit der Publikation von Tagliacozzi im Jahr 1597 [4] hatte sich die Herangehensweise an medizinische Probleme auf der Basis eines naturwissenschaftlich orientierten Krankheitskonzeptes ganz grundsätzlich gewandelt. Die Bereiche Chirurgie und Medizin hatten sich einander stark angenähert und gingen Hand in Hand. Krankheiten wurden in neuen Systemen erfasst, die auf der Idee einer Gewebelehre und einem breiten Vergleich an einer Vielzahl von Patienten beruhten. Als dermatologisches Beispiel sei an die 2. Wiener Medizinische Schule mit Ferdinand von Hebra (1816 - 1880) erinnert, der 1845 seinen „Versuch einer auf pathologischen Anatomie gegründeten Einteilung der Hautkrankheiten“ veröffentlichte. Von Hebra dachte zwar therapeutisch noch mehrheitlich im Sinne der Säftelehre, wie die plastischen Chirurgen ja auch, aber gleichzeitig wurden Therapien und Eingriffe zum wissenschaftlichen Experimentierfeld.

Schon bald darauf erklärte Claude Bernard (1813-1878) mit der Publikation des Werkes „Introduction à l'étude de la médicine experimentale“ im Jahr 1865 die experimentelle Forschung zur Grundlage der Medizin.

Neue Aufgaben an die Adresse der Ärzte sind Ausdruck des gewandelten Denkstils in der Medizin. Die Haut musste physiologisch untersucht werden und experimentelle Erfahrungen wurden gefordert. Der Arzt hatte neu die Legitimation, ja sogar die Pflicht, Versuche durchzuführen, die Lebenskräfte zu lenken, um in seltenen Fällen dadurch sogar die Natur zu übertreffen. Gut bekannt sind die Versuche des englischen Chirurgen John Hunter (1728-1793) über die Vulnerabilität und Reproduktionsfähigkeit von Geweben in Tieren im Vergleich zum Menschen. So versuchte Hunter bereits 1746 bei Hähnen Sporen und Zähne auf Kämme zu verpflanzen. Aus heutiger Sicht ethisch problematisch war Dieffenbachs Versuch, bei einer Frau mit einer linksseitigen Anästhesie gesunde Hautstücke nach unterschiedlicher Vorbehandlung vom Oberarm gegeneinander auszutauschen. Eines der Stücke verwandelte sich vollständig, das andere teilweise in Eiter und es kam zu größeren Wunden. Dennoch konnte Dieffenbach an einer Stelle zeigen, dass eine organische Verbindung entstanden war. Leider riss er dieses letzte verbleibende Hautstück bei einem Verbandswechsel schließlich auch noch ab, sodass die rein experimentelle Transplantation vollständig misslang.

Von Ammon sah es als eine Pflicht der Ärzte an, über ihre Erfahrungen Mitteilung zu machen. Erst wenn eine größere Menge Beobachtungen veröffentlicht und gesammelt sei, werde sich der wahre Nutzen der plastischen Chirurgie zeigen. Und Zeis hielt fest, dass das Resultat einer plastischen Operation umso besser sei, je mehr die physiologischen und pathologischen Prozesse durch den Arzt im Voraus berechnet und geleitet würden.

\section{Plastische Chirurgie und Kosmetik \\ $\nabla$}

Neben dem naturwissenschaftlichen Konzept spielte aber auch die Haltung gegenüber der Bedeutung des ästhetisch normalen Körpers eine neue Rolle.

Die Kosmetik im Sinne einer „Schönheitschirurgie“ wurde zwar von den Medizinern zumindest in den Publikationen verpönt, 
die Korrektur von abnormen Verstümmelungen dagegen als Indikation für plastisch-chirurgische Eingriffe akzeptiert.

Die Autoren erwähnen die „kosmetische“ Motivation der Patienten in zweierlei Hinsicht. Einerseits war das große Bedürfnis des Leidenden nach einer kosmetischen Korrektur eine hilfreiche Voraussetzung, denn der Patient musste sich auf die Operation freuen und durfte sich nicht vor dem Schmerz fürchten.

„Bei der Operation benehmen sich die Kranken meist heldenmüthig. Sie brauchen nicht gehalten zu werden, man hört keine Klage, nicht einen Ton des Schmerzes von ihnen, obwohl die Durchschneidung der Haut, die Verletzung so vieler Nerven des Gesichtes, auch dem weniger Empfindlichen bestimmt grosse Schmerzen verursacht, woraus diese Kranken wohl nach der Operation im freudigen Gefühle ihres Muthes und ihrer Heilung kein Hehl mehr machen.“ [2]. Mit verzagtem Gemüth und Depression sei die Prognose dagegen sehr schlecht. Auch von Ammon zitiert Dieffenbach: „So unmoralisch dem strengen Beurtheiler die Eitelkeit solcher Kranken erscheinen mag, so günstig ist sie für den Operateur, denn Kranke der Art ertragen oft mit beispiellosem Muthe die schwierigsten und schmerzhaftesten operativen Eingriffe.“

Abgesehen von diesen praktischen Argumenten hat aber auch ein Wandel in der Gewichtung der körperlichen Schönheit stattgefunden. In den Anstandsbüchern der frühen Neuzeit wird nicht nur das Benehmen, sondern auch das Aussehen beschreiben. Wünschenswert sei eine harmonische physische Gestalt, fern von all jenen Extremen, die eine „negative Verwunderung“ hervorrufen könnten, wie dies bei monströsen Abweichungen der Fall sei. Wertungen aufgrund des Aussehens zogen schwerwiegende soziale Konsequenzen nach sich: So wurde ein Mensch mit eingesunkener Nase als Syphilitiker stigmatisiert und ihm wurden Unmoral, Degeneration und Verblödung unterstellt. Die plastischen Chirurgen sahen als ihre moralische Pflicht an, den durch ihr Aussehen sozial schwer beeinträchtigten Menschen zu helfen. Schon von Graefe schrieb 1818, dass der Verlust der Nase nicht mehr als Strafe und Zeichen für Unmoral zu gelten habe. Unter mehreren Beispielen berichtet Dieffenbach mit eindrücklicher Betonung seiner persönlichen Emotionen, wie er einer Frau mit durch Lupus zerstörtem Gesicht schließlich wieder ermöglichen konnte, ein Theater zu besuchen.

Zusammenfassend möchte ich das Schlusswort von Ammons nochmals ausführlicher zitieren, in welchem er die dargestellten Begründungen selbst aufzählt:
„Die plastische Chirurgie verdankt die grossen Fortschritte, welche sie in unsern Tagen gemacht hat, offenbar der physiologischen Richtung, die man ihr gegeben. Durch sie haben wir eine genaue und naturgemässe Kenntniss von der Transplantationsfähigkeit der Haute gewonnen: durch sie ist die Nachbehandlung eine wahrhaft rationelle geworden und [...]. So gewährt die plastische Chirurgie, von alten Vorurtheilen befreit, und kühn und kräftig gehandhabt, jetzt grosse Resultate, welche nicht etwa blos von kosmetischem Werthe sind, sondern auch moralischen Einfluss auf viele unserer leiden Mitmenschen üben und sehr bedeutenden therapeutischen Nutzen bei manchen bisher für unheilbar gehaltenen Krankheiten gewähren.“

\section{Abstract}

\section{0 - The Time of Prosperity of Plastic Surgery: Concepts of Disease and their Influence on Surgery} $\nabla$

In 1838 and 1842 Eduard Zeis and Friedrich August von Ammon, both practicing surgeons in Dresden, published their books about "plastic surgery“. They were convinced that they had reached the height of their discipline. But anesthesia and asepsis have not been discovered, yet. This indicates that the classical history of a (technical) progress needs to be completed with aspects of concepts of diseases and the social impact of cosmetic surgery, as they had a direct influence on the thought style of the surgeons.

\section{Literatur}

1 von Ammon FA. Die Plastische Chirurgie nach ihren bisherigen Leistungen kritisch dargestellt. Berlin: Reimer, 1842

2 Zeis E. Handbuch der plastischen Chirurgie. Berlin: Reimer, 1838

3 Dieffenbach JF. Die operative Chirurgie. Leipzig: Grockhaus, 1845 und 1848

4 Tagliacozzi G. De Curtorum Chirurgia per insitionem. Venedig: Libri duo, 1597

5 Blandin P-F. L'Autoplastie ou restauration des parties du corps. Paris: Bermer Baillière, 1836

6 Gadebusch BM. Medizinische Ästhetik. München: Wilhelm Fink, 2005

7 Gilman SL. Making the body beautiful. Princeton: Princeton University Press, 1999 